\title{
Power Characteristics of a Screw Agitator in a Tube
}

F. Rieger

Screw agitators rotating in tubes are very efficient tools for mixing and pumping viscous liquids. The power characteristic of the agitator-tube assembly must be known to enable its power consumption in a given configuration to be calculated. The dimensionless power characteristic is described by Eq. (6). An estimate of power consumption from the power characteristic is schematically shown in Fig. 1. The dependence of the coefficients in Eq. (6) on the Reynolds number is shown in Fig. 5. The power characteristics for selected Reynolds number values are shown in Figs. 6-9.

Keywords: power, power characteristic, screw agitator, pump.

\section{Introduction}

Screw agitators rotating in tubes are very efficient tools for mixing and pumping viscous liquids. They are also suitable for cases where the viscosity of the liquid changes during operation. The power characteristic of the agitator in the tube must be known to enable its power consumption in a given configuration to be calculated. An estimate of power consumption $P$ from the power characteristic is schematically shown in Fig. 1, where the specific energy $e$, for which the power is determined, is given by the intersection of the pumping characteristic of the screw and the hydraulic characteristic of the system (dependencies of flow rate $Q$ on specific energy).

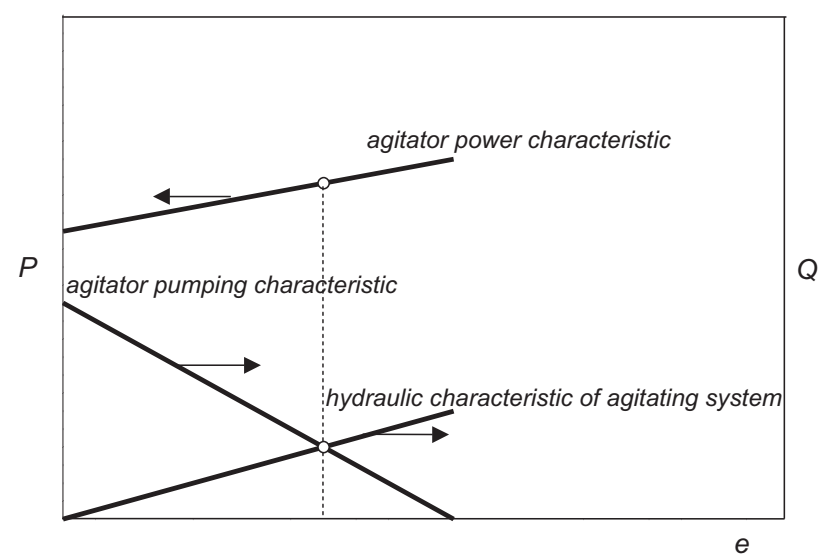

Fig.1: Estimation of power consumption from operating characteristics (creeping flow region)

The information available in the literature on the power consumption of screws concerns mainly the creeping flow regime (screws in extruders). However, screw agitators are often used in transition and eventually turbulent regimes. A method for calculating the power characteristics of screw agitators in a creeping flow regime was proposed in [1]. The power characteristics at higher Reynolds number values must be determined experimentally. An experimental method based on measurements in several configurations was reported previously [2]. However, it should be noted that it is difficult to keep the same geometrical parameters (especially clearance between tube and screw) in different configurations. Therefore, a new method based on measurement in a single configuration was used [3], [4].

\section{Theoretical}

The power characteristic is the dependence of power consumption $P$ on specific energy $e$. Applying inspection analysis of the governing equations, the following relationship for the dimensionless power characteristic was proposed in [2]

$$
P^{*}=P^{*}\left(e^{*}, \mathrm{Re}\right)
$$

where dimensionless power

$$
P^{*}=P / \mu N^{2} D^{3}
$$

dimensionless specific energy

$$
e^{*}=e / v N
$$

and Reynolds number

$$
\operatorname{Re}=N D^{2} / v
$$

In the creeping flow regime Eq.(1) reduces to

$$
P^{*}=P^{*}\left(e^{*}\right)
$$

and the dimensionless power characteristic is linear

$$
P^{*}=c+a e^{*}
$$

At higher Reynolds number values the power characteristics are generally non-linear. However, the dependence of dimensionless power on dimensionless specific energy is not very strong and decreases with increasing Reynolds number, as it is shown Fig. 2, based on the experimental results

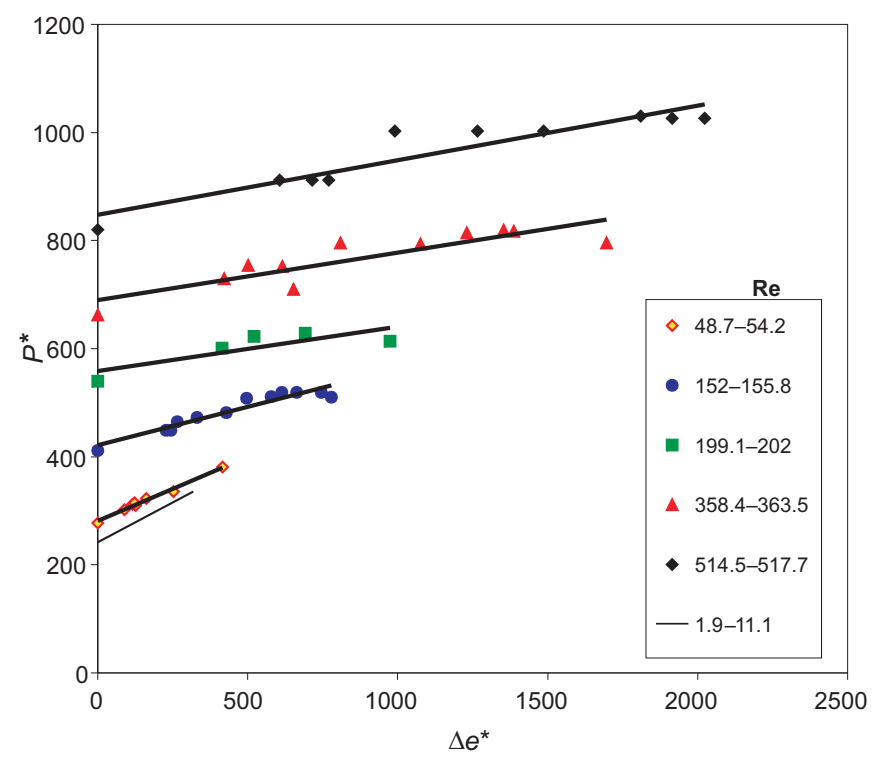

Fig. 2: Power characteristics at different Reynolds number values 
presented in [3]. For this reason we may approximate the dependence of dimensionless power on dimensionless specific energy at given Reynolds number values also by a straight line (6), with coefficients $c$ and a dependent on the Reynolds number. Two pairs of $P^{*}$ and $e^{*}$ values are sufficient for the design of these straight lines. These two points can be represented by the asymptotic power characteristics at minimum and maximum specific energy presented in [4]. Inserting in Eq.(6) we can write

$$
\begin{aligned}
& P_{\text {min }}^{*}=c+a e_{\text {min }}^{*} \\
& P_{\text {max }}^{*}=c+a e_{\text {max }}^{*}
\end{aligned}
$$

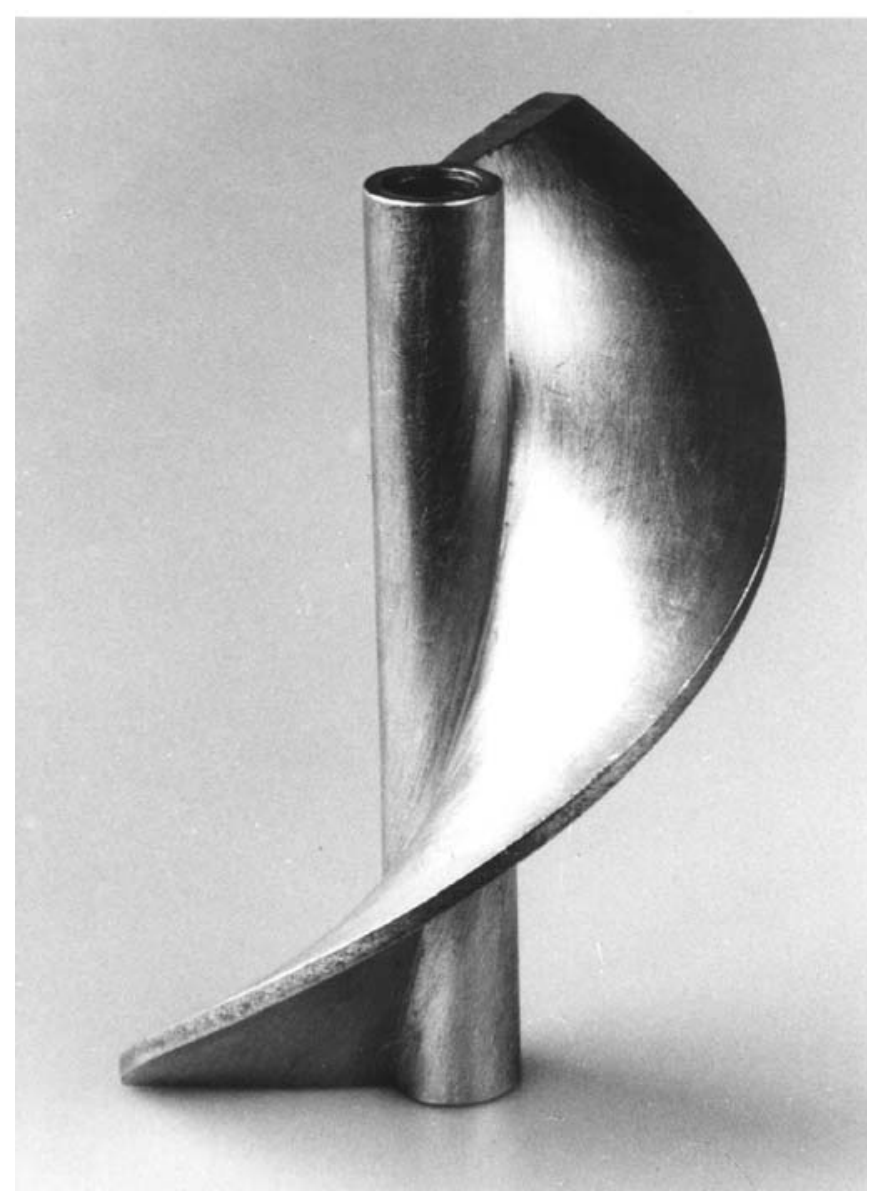

Fig. 3: Screw agitator used in experiments
The value of the dimensionless minimum specific energy $e_{\text {min }}^{*}$ was calculated (in the creeping flow region - see [5], in the turbulent region - see [6]), the value of the dimensionless maximum specific energy $e_{\max }^{*}$ was determined experimentally (see [7] or [3]).

Substracting equations above we obtain the following equation for

$$
a=\left(P_{\max }^{*}-P_{\min }^{*}\right) /\left(e_{\max }^{*}-e_{\min }^{*}\right)
$$

Coefficient $c$ can be expressed from (8)

$$
c=P_{\max }^{*}-a e_{\max }^{*}
$$

\section{Results and discussion}

The procedure proposed above was applied for a screw agitator with the following parameters: pitch $s=2 \mathrm{D}$, root diameter $d=0.2 \mathrm{D}$ and length $L=1.4 \mathrm{D}$ - see Fig. 3 .

The asymptotic power characteristics for this agitator obtained experimentally and reported in [4] are presented in Fig. 4.

The characteristic at minimum specific energy was measured in a relatively large vessel with low hydraulic resistance. The characteristic at maximum specific energy (zero pumping capacity) was attained covering the draught tube. Using the values of dimensionless power $P^{*}=P_{0}$ Re obtained from Fig. 4, the values of coefficients $a$ and $c$ were calculated from Eqs.(9) and (10). The dependencies of $a, c$ and $c / \operatorname{Re}$ on the Reynolds number are presented in Fig. 5. From this figure it follows that in the creeping flow regime values $a$ and $c$ are constant, and in the turbulent region values $a$ and $c /$ Re are constant. For this reason the equation of dimensionless power characteristic (6) in a turbulent region transforms to

$$
P o=c_{\mathrm{t}}+a e^{+}
$$

where $c_{\mathrm{t}}=c /$ Re. Equation (11) is recommended for standard pumps used for pumping low viscosity liquids. Fig. 5 also shows that the value of a in a creeping flow regime is greater than the corresponding value in a turbulent region, which means that the dependence of dimensionless power on specific energy (hydraulic resistance of the system) is less pronounced in a turbulent region. Using the coefficients

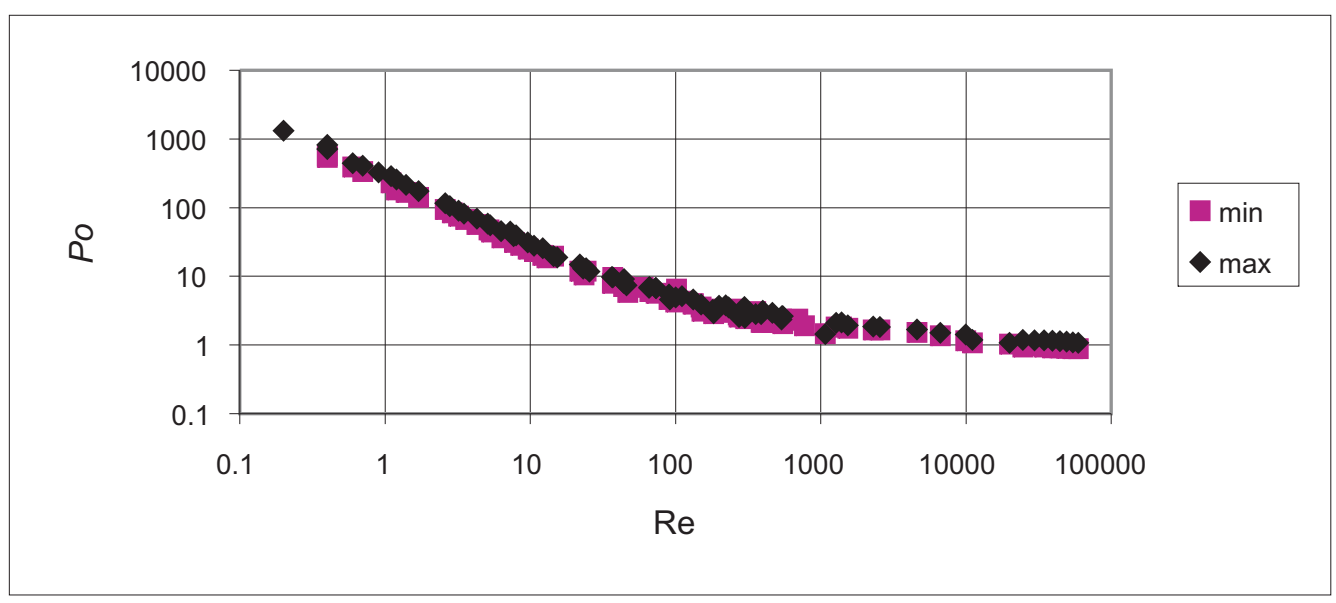

Fig. 4: Asymptotic power curves of the screw agitator 
depicted in Fig. 5, the power characteristics for selected values of Reynolds number can be obtained.

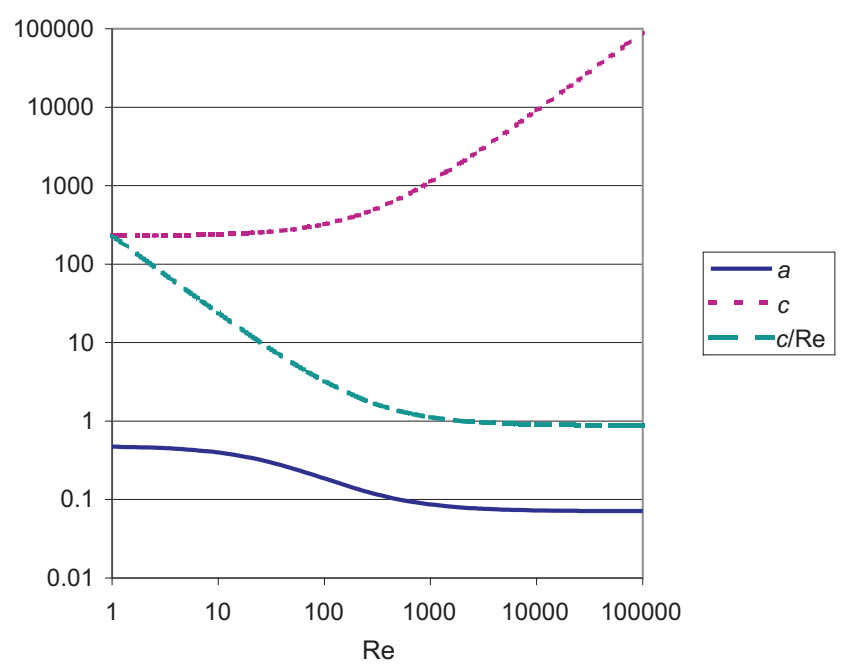

Fig. 5: Dependence of dimensionless coefficients on Reynolds number

Power characteristics in the form of Eq. (6) for a creeping flow region and $\mathrm{Re}=100$ are shown in Fig. 6 .

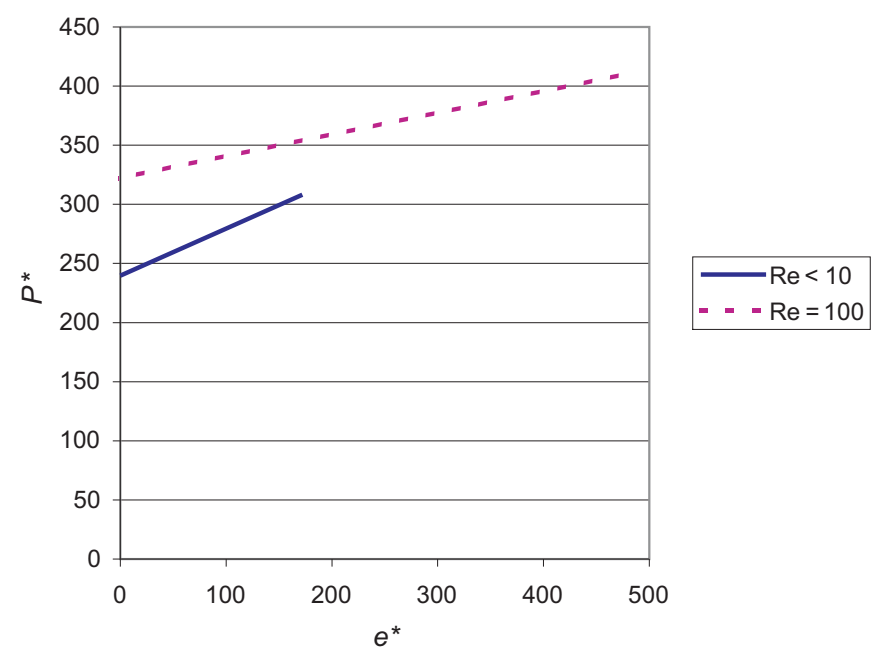

Fig. 6: Power characteristics at small Reynolds number values

Power characteristics in the form of Eq. (11) for $\mathrm{Re}=1000$ and a turbulent region are shown in Fig. 7.

Using the pumping characteristics presented in [8] the power characteristics can be expressed in an alternative form, more frequently used in literature on pumps (se e.g. [9]), with dimensionless flow rate $Q^{*}$ on the axis of the abscissas shown in Figs. 8 and 9.

\section{Acknowledgement}

This research was supported by the Grant Agency of the Czech Republic under Grant No.101/99/0638.

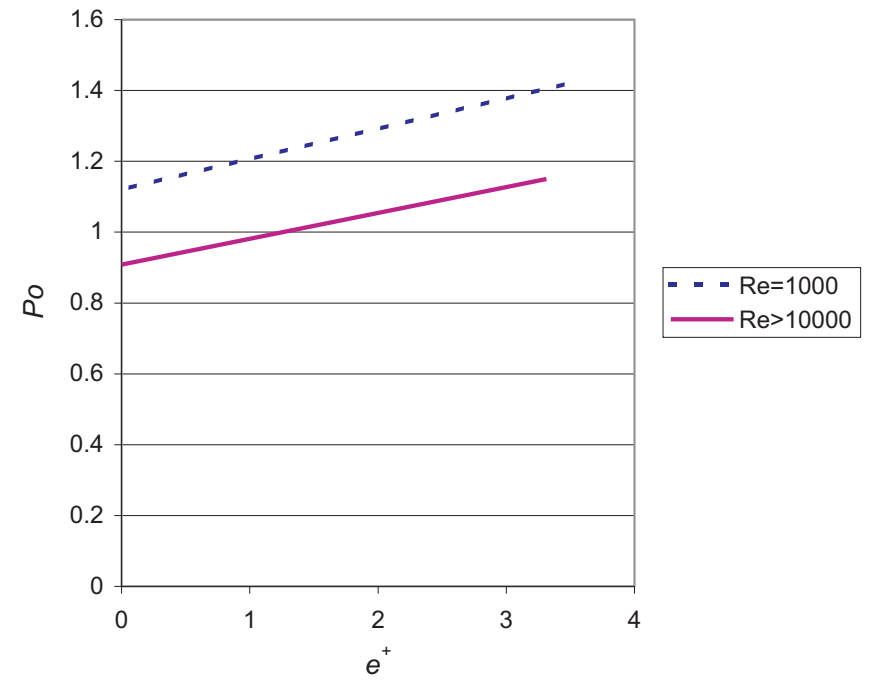

Fig. 7: Power characteristics at high Reynolds number values

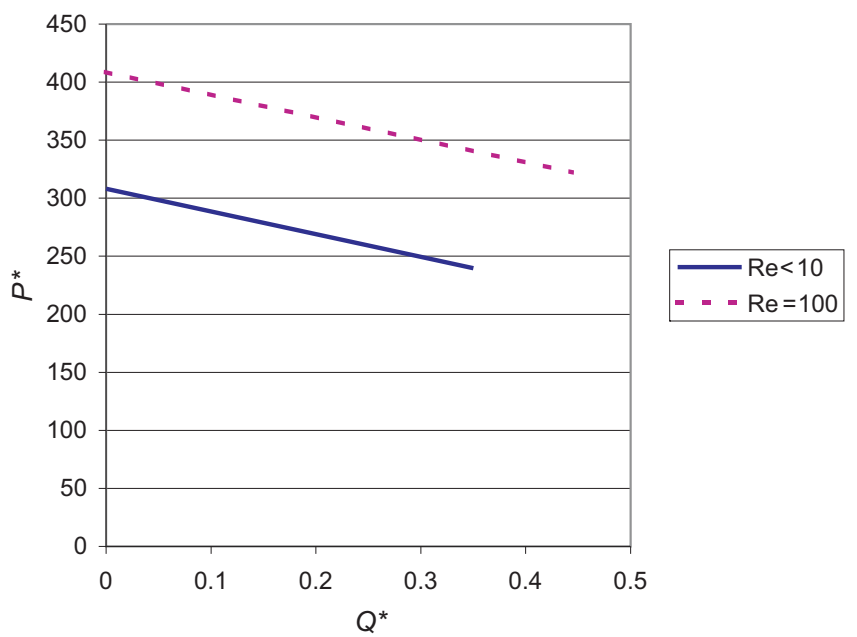

Fig. 8: Power characteristics at small Reynolds number values

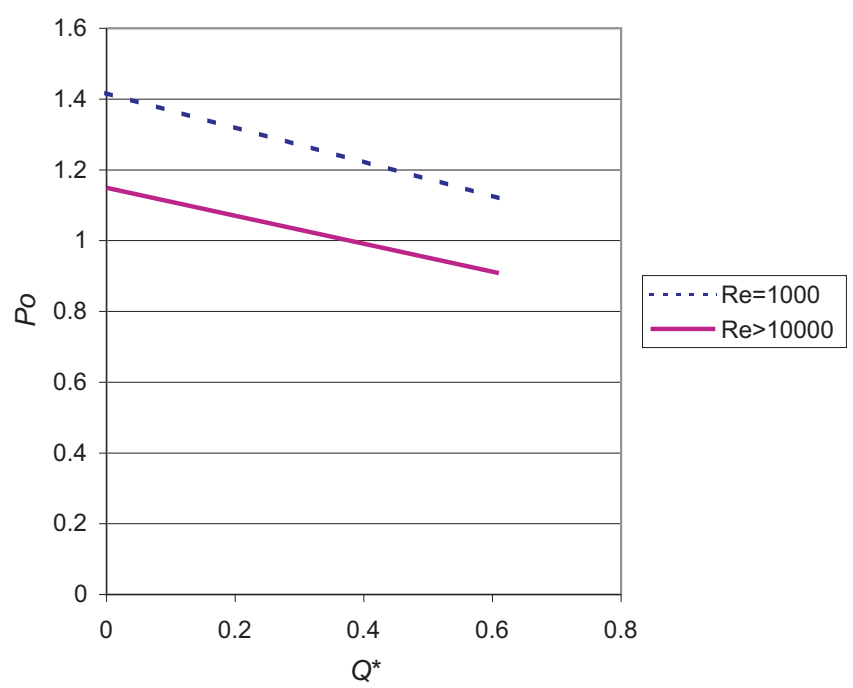

Fig. 9: Power characteristics at high Reynolds number values 


\section{Symbols}

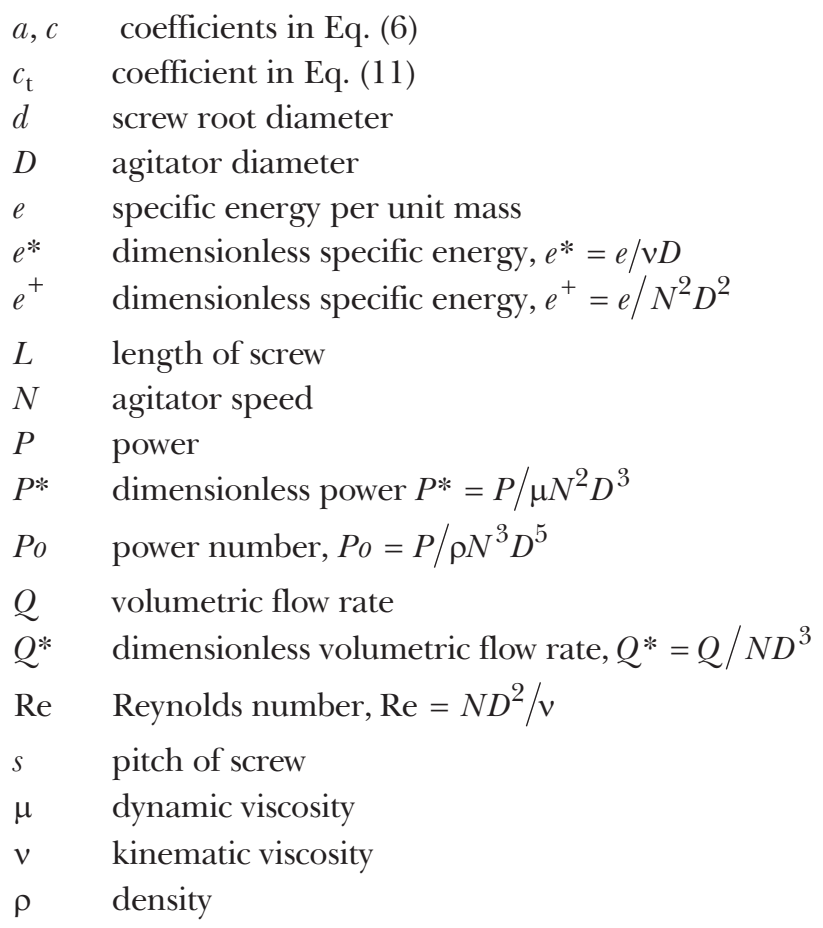

\section{References}

[1] Rieger, F.: The Power Input of Screw Rotors and Agitators. Collect. Czech. Chem. Commun. 54, 1989, pp. 1575-1588

[2] Rieger, F., Weiserová, H.: Determination of Operating Characteristics of Agitators in Tubes. Chem. Eng. Technol. 16, 1993, pp. 172-179
[3] Jirout, T., Brož, J., Rieger, F.: Measurement of Power Characteristics of Screw Agitators in a Tube. Proceedings of Czech Mixing Conference, Techmix, Brno 2000 (CDrom, in Czech)

[4] Rieger, F.: Measurement of Asymptotic Power Characteristics of Screw Agitators in a Tube. CHISA Conference, Czech Society of Chemical Engineering, Srní 1999 (CDrom, in Czech)

[5] Rieger, F.: Power Characteristics of Screw Agitators in Creeping Flow Region. Proceedings of SSCHI Conference, Slovak Society of Chemical Engineering, Tatranske Matliare 2000 (CDrom, in Czech)

[6] Blenke, H., Bohner, K., Hirner, W.: Druckverlust bei der $180^{\circ}$ - Stromungsumlenkung im Schlaufenreaktor. Verfahrenstechnik 3, 1969, pp. 444-452

[7] Rieger, F.: Pumping Characteristics of a Screw Agitator in a Tube. Chem. Eng. J. 66, 1997, pp. 73-77

[8] Sedláček, L., Rieger, F.: Influence of Geometry on Pumping Characteristics of Screw Agitators. Collect. Czech. Chem. Commun. 62, 1997, pp. 1871-1878.

[9] Bláha, J., Brada, K.: Pump Handbook. Prague, ČVUT Publishing House 1997 (in Czech)

Prof. Ing. František Rieger, DrSc. e-mail: rieger@fsid.cvut.cz

Department of Process Engineering

Czech Technical University in Prague

Faculty of Mechanical Engineering

Technická 4, 16607 Praha 6, Czech Republic 